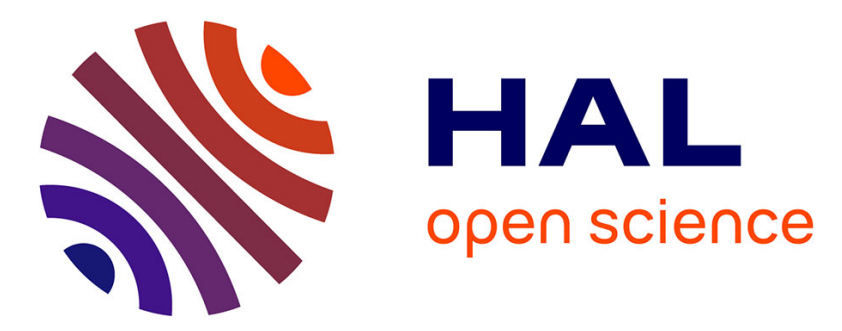

\title{
L'agrandissement de Paris en 1860 : un projet controversé
}

Nathalie Montel

\section{To cite this version:}

Nathalie Montel. L'agrandissement de Paris en 1860: un projet controversé. Bourillon Florence et Fourcaud Annie (éd.). Agrandir Paris (1860 -1970), Publications de la Sorbonne/Comité d'histoire de la Ville de Paris, pp.99-111, 2012, Histoire contemporaine, 978-2859446956. hal-00739392

\section{HAL Id: hal-00739392 \\ https://hal-enpc.archives-ouvertes.fr/hal-00739392}

Submitted on 4 Feb 2016

HAL is a multi-disciplinary open access archive for the deposit and dissemination of scientific research documents, whether they are published or not. The documents may come from teaching and research institutions in France or abroad, or from public or private research centers.
L'archive ouverte pluridisciplinaire HAL, est destinée au dépôt et à la diffusion de documents scientifiques de niveau recherche, publiés ou non, émanant des établissements d'enseignement et de recherche français ou étrangers, des laboratoires publics ou privés.

\section{(ㅇ)(1) $\$$}

Distributed under a Creative Commons Attribution - NonCommercial - NoDerivatives| 4.0 


\section{L’agrandissement de Paris en 1860 : un projet controversé}

Nathalie MONTEL - Université Paris-Est, LATTS (Laboratoire Techniques, Territoires et Sociétés)

Dès le mois de janvier 1860, en application de la loi du 16 juin 1859 qui déplace aux fortifications les frontières de la capitale, la démolition du mur des fermiers généraux commence. En dépit de leur disparition matérielle, ces anciens contours de la capitale laisse dans le paysage urbain une empreinte durable, qui n'est pas seulement perceptible dans le tracé des boulevards que les terrains libérés ont permis d'ouvrir. Un siècle et demi plus tard, l'ancienne frontière subsiste en effet aussi dans le vocabulaire, à travers l'usage courant d'expressions comme «les arrondissements centraux» ou «les arrondissements périphériques », l'espace couvert par ces derniers correspondant grosso-modo à la zone rattachée à la capitale sous le Second Empire. Si la mémoire des anciennes limites de la capitale reste toujours vivace, en revanche le souvenir des conditions dans lesquelles la décision de 1859 fut prise et de ses motivations s'est, quant à lui, progressivement dissous. L'agrandissement de la capitale en 1860 est rapidement devenu un événement sans histoire, dans les deux sens du terme. C'est lorsque de nouveaux projets d'agrandir Paris sont apparus, soit peu avant la première guerre mondiale, que l'idée de s'intéresser à ce précédent survenu un demi siècle plus tôt a surgi ${ }^{1}$. Ce retour sur le passé a servi à la fois à éclairer les projets urbanistiques alors mis à l'étude et à légitimer la conception d'un plan d'extension de la capitale. Bien que de multiples recherches aient depuis lors contribué à enrichir l'histoire de Paris au XIX ${ }^{\mathrm{e}}$ siècle, l'agrandissement de la ville en 1860 est cependant resté considéré davantage comme une donnée à prendre en compte et une césure chronologique commode dans le grand récit du développement de la capitale que véritablement comme un événement à interroger pour lui-même ${ }^{2}$. Quant aux rares études qui ont abordé l'histoire de la décision prise en 1859, elles ont accrédité l'idée d'une mesure évidente ou naturelle et entériné l'opinion d'une réforme qui faisait l'unanimité ${ }^{3}$. C'était là faire peu de cas des voix dissidentes qui se sont exprimées sur le projet impérial de grand Paris.

Lorsque l'on prend la peine de les écouter, c'est-à-dire qu'on ne se contente pas de recourir aux écrits du préfet Haussmann ou aux discours officiels pour écrire l'histoire du recul des limites de la capitale sous le Second Empire, on s'aperçoit en effet que le projet fut contesté et suscita une vive opposition. La controverse qui opposa les autorités à une partie des habitants fut l'occasion pour chaque camp de faire valoir des arguments qui permettent d'éclairer les enjeux multiples associés à la modification du périmètre de la capitale. Avant d'évoquer les motifs et les modalités de cette opposition au déplacement des frontières communales de Paris, il est nécessaire de revenir sur la loi du 16 juin 1859 pour en analyser la teneur et mettre en lumière les diverses facettes de la réforme qu'elle met en oeuvre. Agrandir

\footnotetext{
${ }^{1}$ Préfecture du département de la Seine, Commission d'extension de Paris. Aperçu historique, Paris, Imprimerie Chaix, 1913.

${ }^{2}$ C'est par exemple le point de vue implicite des études qui font de 1860 l'année de naissance de la banlieue parisienne ou qui organise l'enquête approfondie de Gérard Jacquemet : Belleville au XIX siècle du faubourg à la ville, Paris, Éditions de l'École des hautes études en sciences sociales et Jean Touzot, 1984.

${ }^{3}$ Ce constat général mériterait, cependant, d'être quelque peu nuancé. Il faudrait par exemple citer la thèse que Jeanne Gaillard a consacrée à Paris sous le Second Empire. Bien qu'elle aborde le sujet de l'extension du territoire de la capitale en 1860 , cette recherche la considère toutefois avant tout sous l'angle restreint d'une question vive à l'époque de son écriture, à savoir les relations entre urbanisation et industrialisation : J. Gaillard, Paris, la ville (1852-1870), Paris, L'Harmattan, 1997. Pour un examen plus approfondi de ces analyses à caractère téléologique, au surplus peu soucieuses de la critique historique des sources qu'elles mobilisent, voir Nathalie Montel, «Chronique d'une mort non annoncée. L'annexion par Paris de sa banlieue en 1860 », Recherches contemporaines, $\mathrm{n}^{\circ}$ 6, 2000-2001, p. 217-254. En se fondant sur l'ensemble des sources disponibles, cet article s'efforce de saisir la pluralité des points de vue en présence et d'écrire une histoire de l'événement qui ne soit pas une simple chambre d'écho aux propos tenus par les pouvoirs publics.
} 
Paris n'est en effet qu'un des aspects, et probablement pas le plus important, de cette loi. La confrontation de deux caricatures du Second Empire représentant l'extension de la capitale permettra enfin d'illustrer en images l'opposition des points de vue en présence et de remettre en question quelques idées reçues ou interprétations hâtives de l'évènement.

\section{La loi du 16 juin 1859 : une réforme spatiale, politique et fiscale}

Le texte législatif du 16 juin 1859 est officiellement baptisé « loi sur l'extension des limites de Paris ». Ce titre ne rend compte que de manière partielle et partiale de son contenu réel, à savoir une réforme territoriale indissociablement administrative, politique et fiscale.

L’intitulé de la loi traduit d'abord un regard parisiano-centré sur la réforme exposée. Loin de concerner exclusivement la capitale, ce texte organise une refonte d'envergure de la géographie administrative du département de la Seine. Il importe donc de situer l'analyse à cette échelle et de ne pas seulement examiner la carte du Paris agrandi. Choisir les fortifications pour nouveau périmètre de la capitale conduit à un doublement de sa superficie, mais il remet aussi en cause les contours de vingt-huit autres communes du département, les limites de sept des huit cantons existant en dehors de Paris et les frontières des trois arrondissements départementaux de la Seine. La construction des fortifications à partir de 1841 s'était, il faut le rappeler, peu embarrassée des limites administratives existantes, l'enceinte défensive ayant été édifiée sur les territoires de vingt communes. La réforme affecte différemment ces vingt-huit communes. Parmi elles, onze sont supprimées et dix-sept autres voient leurs contours redessinés. Au sein des communes qui disparaissent, quatre sont intégralement absorbées par le nouveau Paris - La Villette, Belleville, Vaugirard et Grenelle tandis que sept autres - Auteuil, Passy, Batignolles-Monceau, Montmartre, La Chapelle, Charonne et Bercy - laissent en dehors de la capitale agrandie des portions de territoires. Celles-ci sont rattachées aux communes qui les jouxtent. Les dix-sept autres communes touchées par la réforme territoriale peuvent être réparties en trois groupes. Neuf d'entre elles perdent des parties plus ou moins conséquentes de leurs territoires au profit de Paris - Neuilly, Le Pré Saint-Gervais, Pantin, Saint-Mandé, Ivry, Gentilly, Montrouge, Vanves et Issy -, tandis que quatre, toutes situées au-delà des fortifications, s'agrandissent - Saint-Denis, Boulogne, Montreuil et Charenton-le-pont - et que les quatre dernières à la fois gagnent et cèdent des terrains - Clichy, Saint-Ouen, Aubervilliers et Bagnolet.

Le libellé choisi pour cette loi tend également à masquer la dimension politique de la mesure. En supprimant onze communes, la loi du 16 juin 1859 signe aussi l'acte de décès de onze municipalités. Par son agrandissement, la capitale englobe la population qui vit entre le mur des fermiers généraux et l'enceinte de Thiers, estimée alors à 400000 habitants, et la soumet au régime d'exception qui régit la municipalité parisienne. En devenant parisienne, cette population passe entièrement sous l'administration directe de l'État et la coupe des deux préfets de la Seine, le préfet du département et le préfet de police. Les arrondissements parisiens et la commune de Paris n'ont en effet pas de maire, et les pouvoirs de son conseil municipal sont extrêmement restreints. Les populations des communes supprimées perdent le reste d'autonomie municipale dont elles jouissaient jusque-là, c'est-à-dire la faculté de s'administrer elles-mêmes, de décider d'une partie de leur fiscalité et de l'affectation de leurs ressources financières. Ces droits municipaux, dont les habitants de la zone annexée se trouvent privés, avaient déjà été restreints par une loi du 5 juillet 1855 qui plaçait l'ensemble des communes du département de la Seine sous un régime dérogatoire au régime général, les membres de leur conseil municipal n'étant plus désormais élus mais nommés par le gouvernement. Ce dernier se montrait en effet déjà soucieux de contrôler les populations vivant aux alentours de la capitale, considérées comme potentiellement dangereuses par les pouvoirs et la bourgeoisie. Pour la même raison, la loi du 10 juin 1853 avait autorisé le préfet 
de police à étendre à toutes les communes de la Seine les fonctions qu'il exerçait dans Paris depuis l'arrêté des Consuls du 12 Messidor an VIII'.

Enfin, l'intitulé de la loi passe sous silence sa dimension fiscale. Ce texte ne prononce pas seulement le recul des limites administratives de la capitale d'une enceinte à l'autre. Il étend aussi jusqu'aux fortifications le régime fiscal propre à la ville de Paris. La population vivant entre les deux murs se trouve ainsi désormais assujettie aux contributions directes et indirectes de la capitale, et notamment à l'octroi parisien. Principale ressource financière de la capitale, cet impôt indirect taxe la plupart des produits qui entrent dans Paris, et en particulier les denrées alimentaires de première nécessité. Sur les territoires à englober, des octrois existent mais leurs tarifs sont plus faibles et concernent moins de produits. C'est d'ailleurs la raison pour laquelle les populations les plus modestes ont choisi d'aller vivre au-delà du mur des fermiers généraux, y trouvant des loyers moins chers et une vie à meilleur marché. Cette migration explique en partie un phénomène mis en évidence par les résultats des recensements effectués en 1851 et 1856 et qui a retenu l'attention des pouvoirs politiques, à savoir que ces communes limitrophes de Paris connaissaient les plus forts taux de croissance démographique du département. Elles ont notamment accueilli les habitants de l'ancien Paris chassés de leur logement par les travaux qui s'exécutent depuis quelques années dans le centre de la capitale. C'est également pour se soustraire aux taxes de l'octroi parisien sur les matières premières, qu'un certain nombre d'activités industrielles ont choisi de s'implanter au-delà des barrières des fermiers généraux. La loi de juin 1859 prévoit quelques dispositions transitoires pour adoucir les conséquences de ce changement de régime fiscal. Ces aménagements visent d'une part la population la plus aisée et d'autre part la grande industrie et le commerce en gros. S'agissant en effet de l'augmentation des contributions directes, dont les taux croissent en fonction de l'importance de la population, un moratoire de cinq années est accordé à ceux qui les payent, c'est-à-dire aux plus fortunés. Quant au nouveau régime de l'octroi, qui doit s'appliquer dès le $1^{\text {er }}$ janvier 1860 , des délais et facilités sont consentis. Ils concernent exclusivement les établissements du commerce en gros, qui se voient proposer de bénéficier pendant dix ans de l'entrepôt à domicile, et les usines, pour lesquelles le projet prévoit de différer de sept ans la hausse des taxes sur la houille et les matières premières employées. Des protestations s'élèvent contre chacun de ces différents aspects de la réforme.

\section{Formes et natures de l'opposition au Grand Paris de $1860^{5}$}

Dès son annonce, le projet d'agrandir Paris suscite une opposition, dans un contexte où protester est tout sauf une démarche anodine. Le Second Empire est en effet marqué par une réduction considérable des libertés publiques et par une surveillance des écrits et des paroles. La presse, l'imprimerie et la librairie sont étroitement contrôlées depuis 1852. Les associations ou les réunions publiques sont, elles aussi, strictement limitées. À fin de l'année 1858, lorsque le préfet Haussmann laisse entendre puis confirme qu'il prépare le déplacement les limites de la capitale, se clôt tout juste une période de " crispation autoritaire » du régime. Ayant fait suite à l'attentat d'Orsini contre l'Empereur, elle s'est traduite par le vote de la loi de sûreté générale du 19 février 1858 qui a restreint encore davantage la liberté d'expression et durci la répression.

\footnotetext{
4 Maryvonne Bernard, «La réorganisation de la police sous le Second Empire (1851-1858): «des bras infatigables ", in Philippe Vigier (éd.), Maintien de l'ordre et polices en France et en Europe au XIX ${ }^{\circ}$ siècle, Paris, Créaphis, p. 120-135.

${ }^{5}$ Cette partie de la communication s'appuie sur un travail en cours de publication dont il reprend quelques résultats. Voir N. Montel, Le projet de Grand Paris impérial, au prisme des archives de l'enquête publique de 1859, Rennes, Comité d'histoire de la ville de Paris/Presses universitaires de Rennes, 2011, à paraître.
} 
L'opposition au projet de loi se manifeste principalement de deux manières. Tout d'abord, par le biais de quelques articles de presse qui tentent d'analyser les enjeux de la mesure annoncée ${ }^{6}$ et la circulation dans l'agglomération parisienne de brochures diffusées par des opposants hostiles à la perte d'autonomie municipale de leur commune et à l'extension du régime parisien de l'octroi ${ }^{7}$. Mais elle s'exprime également, de façon moins attendue, peu de temps après au travers de la procédure administrative de consultation organisée par la préfecture de la Seine. Une loi du 18 juillet 1837 sur l'administration communale prévoyait en effet que les modifications des contours des communes devaient être prononcées par une loi, donc passer devant les Chambres, mais également être l'objet au préalable d'une consultation des habitants et des différentes assemblées représentatives ${ }^{8}$. Les habitants des vingt-huit communes et douze arrondissements parisiens concernés, soit plus d'un million et demi de personnes, ont été informés par le journal officiel, mais surtout par des affiches ou les crieurs publics municipaux de la tenue d'une enquête publique, du 13 au 27 février 1859, et de la mise à disposition de documents sur le projet de Grand Paris concocté par les autorités. Environ quatre mille cinq cents personnes ont laissé une trace de leur opinion sur des registres ouverts à cette intention dans les mairies ${ }^{9}$. Dans une écrasante majorité, ces déposants ont exprimé un désaccord à l'égard du projet qui leur était soumis.

La géographie des dépositions montre sans surprise que c'est dans la zone annexée que les plaintes sont les plus nombreuses. Les motifs des oppositions au projet enregistrées au cours de l'enquête publique sont variés. Certaines protestations concernent les nouveaux découpages territoriaux, les limites d'arrondissements ou de communes, jugées peu pertinentes, arbitraires voire très pénalisantes pour l'avenir. Des habitants de Billancourt, par exemple, ne veulent pas être rattachés à Boulogne ${ }^{10}$, tandis que des Bellevillois contestent la scission de leur ancienne commune en deux arrondissements parisiens distincts, les actuels $19^{\mathrm{e}}$ et $20^{\mathrm{e} 11}$. D'autres remarquent que dans le nouveau Paris les mairies ne seront plus, dans l'immédiat au moins, au centre des circonscriptions et se plaignent de l'allongement des trajets que les nouveaux-nés auront à effectuer au péril de leur vie pour être présentés à l'officier d'état civil. Les habitants des communes sérieusement amputées au profit de la capitale, comme Montrouge, Saint-Mandé ou Le Pré Saint-Gervais, déplorent quant à eux de voir leur territoire perdre des contribuables et se plaignent d'être dépouillés de leur mairie, de leurs écoles ou de leurs commerces. Les doléances enregistrées viennent aussi rappeler que Paris à cette époque était encore loin d'être à l'étroit dans ses limites de 1790 ou que le futur empereur avait pris l'engagement écrit en novembre 1852 de ne pas déplacer les barrières de l'octroi parisien ${ }^{12}$. Mais les revendications exprimées s'attachent surtout à dénoncer les effets funestes de l'augmentation de l'octroi, à la fois sur l'activité industrielle qui s'est développée

\footnotetext{
${ }^{6}$ Le Constitutionnel, 17 et 20 décembre 1858. La Presse, 27 décembre 1858, 11 et 17 janvier 1859.

7 Albert Perrin, Deuxième étude administrative. Des intérêts de la banlieue de Paris, examen d'un projet d'annexion, Paris, Imprimerie Chaix, 1858 ; V. B., propriétaire intra et extra muros, De l'agrandissement de Paris et de son opportunité et des moyens d'y parvenir, Paris, Imprimerie et lithographie de Renou et Maulde, 1858 ; Note du conseil municipal de la commune de La Villette sur le projet d'extension de la ville de Paris jusqu'aux fortifications (7 février 1859), Paris, Imp. de Renou et Maulde, s. d. ; Observations présentées au nom des habitants, propriétaires et commerçants de la commune d'Ivry (Seine) au sujet de l'annexion projetée des communes suburbaines à la capitale, par la suppression du mur d'enceinte et le reculement de la limite de l'octroi jusqu'à la ligne des fortification (5 février 1859), Paris, Impr. de E. Brière, s. d.

${ }^{8}$ Sur les modalités particulières de ces diverses consultations et l'organisation pratique de l'enquête publique de février 1859, mais aussi l'analyse des écrits recueillis et la reproduction d'un certain nombre de dires consignés sur les registres, voir N. Montel, Le Grand Paris de 1860..., op. cit.

${ }^{9} \mathrm{AN}, \mathrm{F}^{2}$ II Seine 36 et 37.

${ }^{10} \mathrm{AN}, \mathrm{F}^{2} \mathrm{II}$ Seine 36, dossier Auteuil. Dires annexés au registre d'enquête $\mathrm{n}^{\circ} 1,2$ et 3 .

${ }^{11}$ AN, F2II Seine 36, dossier Belleville. Avis du commissaire enquêteur et dire annexé au registre d'enquête $\mathrm{n}^{\circ} 19$.

${ }^{12}$ Le Moniteur universel, $\mathrm{n}^{\circ}$ 324, 19 novembre 1852.
} 
au-delà du mur des fermiers généraux et sur les conditions de vie matérielles des populations les plus modestes qui y résident. Plusieurs déposant craignent que ces dernières soit contraintes, dans un avenir proche, de se réfugier au-delà des fortifications. Certains habitants s'interrogent aussi sur l'affectation des nouvelles ressources que procureront à la ville de Paris ces 400000 nouveaux contribuables et sur les compensations immédiates que les territoires annexés obtiendront en regard des nouvelles impositions auxquelles ils seront soumis. Quelques-uns n'hésitent pas à souligner le vague des promesses d'amélioration de leur sort que les autorités leur font miroiter et remarquent notamment l'inexistence de plan d'aménagement de leurs territoires. D'autres en appellent à une réforme de l'octroi parisien ou au remplacement par une augmentation des impôts directs de cet impôt indirect jugé particulièrement injuste, parce qu'il frappe de la même manière toutes les classes sociales et taxe notamment de façon identique tous les vins, qu'il s'agisse de Château Margaux ou de la piquette que boit le petit peuple ${ }^{13}$. Enfin, s'inscrivant dans une perspective de plus longue durée, un petit nombre estime que le recul des barrières de l'octroi, loin de résoudre les problèmes de l'agglomération parisienne, les déplaceront simplement, dans le temps et dans l'espace. Ils prédisent que les phénomènes observés de part et d'autre du mur des fermiers généraux se reproduiront à l'identique en deçà et au-delà des fortifications.

Appelés à leur tour à se prononcer sur le projet, sept conseils municipaux ont également fait connaître leur désapprobation ${ }^{14}$. Largement passée sous silence par les autorités, cette résistance des municipalités au projet impérial provient des communes de La Villette, Bercy, Ivry, Montrouge, Vaugirard, Grenelle et Auteuil ${ }^{15}$. En dépit de ces oppositions municipales et citadines, la loi fut votée par les Chambres, moyennant l'introduction de modifications mineures au projet présenté aux populations. Les communes récalcitrantes furent ainsi intégrées malgré elles à la capitale. Le combat très inégal qui a vu s'affronter les autorités et les détracteurs de la mesure a laissé derrière lui un grand nombre de traces, des écrits surtout, dont certains viennent d'être évoqués, mais aussi des images.

\section{La controverse en images}

Prolongeant et complétant les discours, des représentations allégoriques de l'agrandissement de la capitale parus dans les journaux de l'époque traduisent les visions contrastées de la décision prise en 1859 et en illustrent les enjeux. Dans la production iconographique disponible, deux caricatures réalisées par un même dessinateur, Charles Vernier (1821-1887), permettent de visualiser à la fois l'argumentation officielle et celle des opposants. Elles ont toutes deux été publiées dans la rubrique «actualités » du Charivari, un quotidien satirique illustré de grande diffusion créé en 1832. Sous le Second Empire, Le Charivari, à l'instar de l'ensemble des journaux, ne peut ignorer les lois répressives sur la presse. La plupart des caricaturistes qui collaborent à ce quotidien, parmi lesquels on peut citer des dessinateurs aujourd'hui davantage connus comme Honoré Daumier (1806-1879) ou Amédée de Noé (1818-1879), dit Cham, s'emparent également du sujet de l'agrandissement de Paris. Tous veillent à éviter les ciseaux de la censure et se préoccupent de ne pas compromettre la survie de la publication par des critiques trop virulentes à l'égard des pouvoirs en place. Replacés dans leur contexte historique, ces dessins se révèlent néanmoins constituer des sources de premier plan pour l'analyse de la réforme de 1860.

\footnotetext{
${ }^{13}$ La référence au Château Margaux se trouve dans Observations présentées..., op. cit..

${ }^{14}$ Dans certaines communes, il fallut recourir au vote. Aux Batignolles, c'est ainsi à une majorité de 23 voix contre 15, que le conseil s'est dit favorable à la mesure. AN, F² II Seine 36, dossier Batignolles-Monceaux. Procès-verbal du conseil municipal, 9 mars 1859.

${ }^{15}$ N. Montel, Le Grand Paris de 1860..., op. cit.
} 


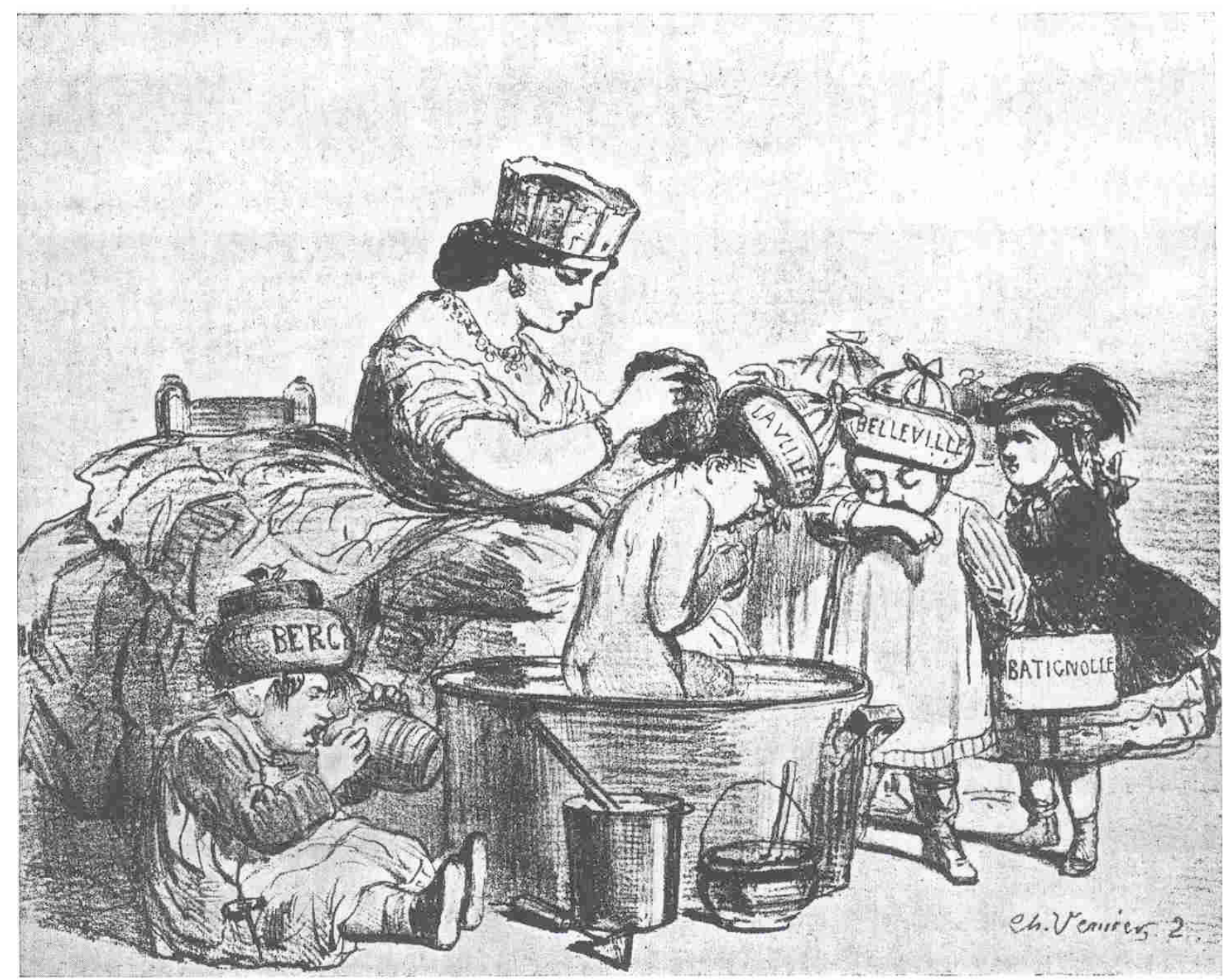

Photo 1 : Charles Vernier, «la bonne ville de Paris et ses nouveaux enfants », Le Charivari, « actualités », 31 janvier 1860, p. 143.

Intitulé « la bonne ville de Paris et ses nouveaux enfants », le premier de ces dessins de presse a paru le 31 janvier 1860, soit un mois après l'entrée en vigueur de la réforme. Les communes du département de la Seine y sont représentées par des personnages. Une femme élégamment vêtue, parée de riches bijoux, porte sur la tête un diadème. Signe de pouvoir et de souveraineté, cette couronne emprunte sa forme aux fortifications et permet d'identifier Paris. Elle est en présence d'une ribambelle d'enfants, deux fillettes et deux garçonnets que l'on distingue nettement et d'autres que l'on devine derrière, dont on n'aperçoit que les bonnets. Les enfants au premier plan portent distinctement les noms de quatre des communes supprimées au $1^{\mathrm{er}}$ janvier 1860 : Bercy, La Villette, Belleville et Batignolles, mais elles sont à leur tour dotés d'attributs qui permettent aux Parisiens de les reconnaître aisément. Assis par terre, à gauche, le petit Bercy possède un tire-bouchon en guise de hochet. Vêtu d'un tablier, il porte un tonnelet à ses lèvres, ces différents objets rappelant que la commune abrite de vastes entrepôts dédiés au commerce des vins et boissons. Au centre de l'image, La Villette est nue au milieu d'une grande bassine cylindrique en fer blanc, qui fait référence très probablement à la présence du bassin de La Villette, point de jonction des canaux parisiens, sur cette commune. À la droite de l'image, se tient la demoiselle des Batignolles, dont la tenue vestimentaire rappelle qu'elle est la plus bourgeoise des quatre communes représentées. Certains Parisiens aisés n'avaient d'ailleurs pas hésité à quitter la capitale pour aller s'installer dans les immeubles élégants qui s'y étaient récemment construits, au grand dam du préfet 
Haussmann qui voyait d'un mauvais œil la concurrence que cette commune faisait aux opérations immobilières en plein essor dans l'ancien Paris ${ }^{16}$.

Le dessinateur a mis l'accent sur un double contraste, d'une part entre un adulte et des personnes mineures, d'autre part entre une femme aisée et des enfants de conditions beaucoup plus modestes, le personnage représentant les Batignolles occupant une position intermédiaire, tant du fait de son âge que de ses vêtements. Cette image rend compte des arguments officiels justifiant que ces communes limitrophes soient englobées dans Paris, mais aussi de la stigmatisation des territoires annexées qui a accompagné l'opération. L'annexion de la zone entre les deux murs était officiellement motivée par le souci de la mettre sur un pied d'égalité avec la capitale, tant sur le plan de la sécurité qu'au point de vue de l'aménagement. Il s'agissait de lui donner à la fois une police et des équipements urbains qui lui auraient fait défaut et dont elles n'auraient pas été capables de se doter par elles-mêmes, c'est-à-dire des agents permettant le maintien de l'ordre mais aussi de larges rues et des réseaux d'eau, d'égouts et de gaz ${ }^{17}$. En choisissant de représenter ces communes par des enfants entourés de jouets, le dessinateur suggère qu'elles manquaient d'autonomie et de maturité, qu'elles avaient besoin d'une tutelle et ne pouvaient pas s'occuper d'elles-mêmes. Il montre également des enfants pauvres, voire indigents, et pas très bien éduqués : le petit Bercy boit du vin, Belleville s'essuie le nez avec le revers son bras et La Villette a besoin d'être lavée, ce qui laisse supposer qu'elle est très sale. La ville de Paris leur prodigue ses soins et en particulier nettoie une ancienne commune de banlieue avec sa grosse éponge ${ }^{18}$. En ce milieu du XIX ${ }^{\mathrm{e}}$ siècle, la lutte contre la saleté des pauvres prend la forme d'un véritable combat pour la bourgeoisie. Celle-ci entend laver les classes laborieuses pour les rendre meilleures, la malpropreté étant à ses yeux étroitement associée au vice et au désordre social $^{19}$. Il y a peut-être aussi dans le choix de Vernier de mettre précisément La Villette au centre de son dessin et de la représenter frissonnante en train de se faire savonner par Paris une allusion au fait qu'elle fut la commune la plus farouchement hostile à son intégration dans la capitale. Quoi qu'il en soit, ce que Vernier met principalement en scène, c'est une relation affectueuse et généreuse, une attitude maternelle et attentionnée de la part de la ville de Paris à l'égard des communes qu'elle a prises dans son giron. Le dessin et son titre reflètent parfaitement les discours officiels en montrant l'action bienfaitrice de la ville de Paris à l'égard de la zone annexée. Le dessinateur serait-il resté totalement sourd aux plaintes et aux critiques portées par ses contemporains sur la mesure d'agrandissement de la capitale ?

\footnotetext{
${ }^{16}$ Commission des circonscriptions administratives, Paris, Charles Mourgues frères, décembre 1857, p. 3.

${ }^{17}$ Delangle, « Rapport à l'Empereur », Le Moniteur universel, 12 février 1859.

${ }^{18}$ On notera la récurrence jusqu'à notre époque de cette volonté exprimée par les autorités de «nettoyer » des communes de banlieues. Seuls les procédés diffèrent.

${ }^{19}$ Voir Julia Csergo, Liberté, égalité, propreté. La morale de l'hygiène au XIX siècle, Paris Fayard, 1988.
} 


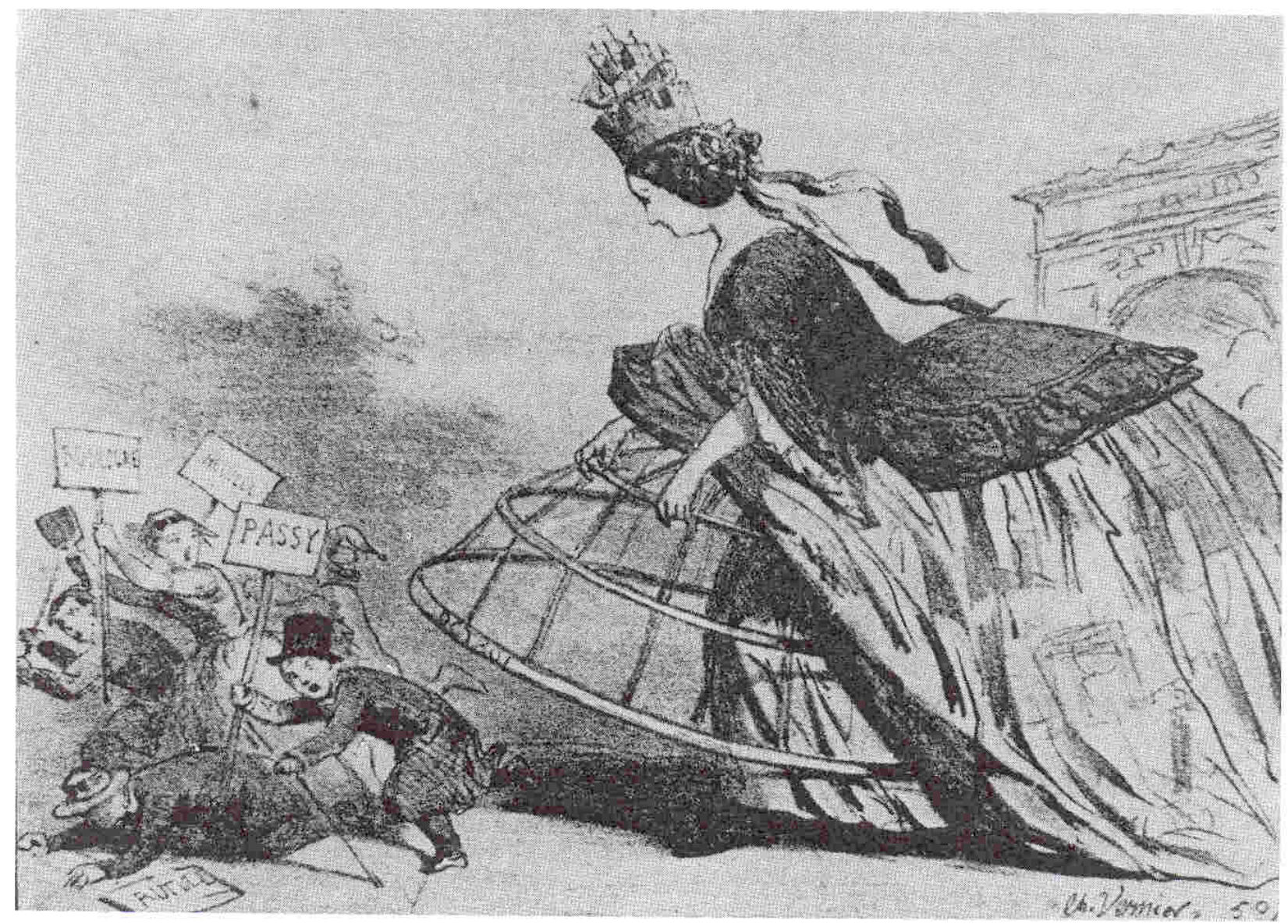

Photo 2 : Charles Vernier, « Paris voulant englober la banlieue », Le Charivari, « actualités », 6 novembre 1858 , p. 576.

Un dessin paru deux mois lus tôt de Vernier prouve qu'il n'en est rien. Intitulé « Paris voulant englober la banlieue », il illustre la contestation qui naît avec l'annonce du projet. La confrontation entre les deux caricatures montre comment, à partir de l'usage de codes et de conventions graphiques très proches, notamment le choix de personnifier les communes et de leur donner des tailles différentes, ces deux caricatures véhiculent des visions opposées de la réforme. On retrouve le style particulier de Charles Vernier mais c'est ici un tout autre point de vue sur la réforme qui est donné à voir. Ce n'est plus l'extension des limites de Paris que met en évidence le dessinateur mais l'annexion par la capitale des communes qui l'environnent. Paris y est déjà représentée par une femme de la bonne société. L'enceinte murée dont elle est coiffée est là surmontée du navire appartenant au blason de la ville. Elle est habillée à la mode, la crinoline cage avec son cerceau ayant fait son apparition en 1856 . Très en vogue durant tout le Second Empire, ces robes à crinoline furent une grande source d'inspiration pour les caricaturistes. Cette fantaisie vestimentaire des femmes de la haute société que la bourgeoisie s'est empressée d'imiter est devenue l'une de leur cible favorite. Si les communes de banlieue sont, ici aussi, représentées au moyen de personnages beaucoup plus petits que Paris, il ne s'agit là plus d'enfants, mais d'adultes. Au premier plan, la commune huppée de Passy est représentée par un homme portant redingote, chapeau haut de forme et se déplaçant avec une canne, accessoire distinctif de la bourgeoisie élégante depuis la monarchie de Juillet. Les pancartes portées par ses voisins révèlent trois autres noms : Boulogne, Neuilly et Auteuil.

La date à laquelle ce dessin a été réalisé importe pour son interprétation. Il est paru le 6 novembre 1858. À ce moment-là, l'annonce n'est pas officielle, mais la rumeur court déjà que l'empereur souhaite agrandir Paris et Haussmann vient de faire savoir officiellement que 
ses services planchent sur le dossier ${ }^{20}$. La population s'attend à ce que la mesure soit annoncée par un décret et sans autres formalités. Elle s'attend surtout à ce qu'Haussmann mette en oeuvre un projet présenté en 1856 à la commission départementale qui consistait à étendre Paris jusqu'à la Seine, c'est-à-dire à agrandir la capitale vers l'ouest uniquement. Le projet de Grand Paris caressé par le préfet était en effet délimité à l'ouest et au sud par le fleuve, au nord par un tronçon des fortifications et par deux lignes de chemin de fer, celle de Saint-Germain, dans la commune de Clichy, et celle du nord, dans la commune de La Chapelle. À en croire certaines affirmations à la vie tenace, les fortifications constituaient des limites évidentes pour l'agrandissement de la capitale et la construction de l'enceinte de Thiers annonçait la décision prise en 1859. Elles ne résistent pas à l'évocation de ce projet soutenu par le préfet. L'extension de la commune de Paris et de son octroi jusqu'à la muraille défensive étaient certes des possibilités, mais en aucun cas des fatalités ${ }^{21}$. Loin d'être inéluctables, ces décisions furent les produits de choix politiques soigneusement pesés ${ }^{22}$. Vernier a soin de montrer que l'action se situe à l'ouest de la capitale, en reproduisant à l'extrême droite de son dessin l'arc de triomphe de l'Étoile. C'est bien à ce projet de Grand Paris délimité par la Seine auquel il fait ici référence. D'où également la présence des communes de Boulogne, sous les traits d'une blanchisseuse aux allures de "Liberté guidant le peuple » brandissant son battoir ${ }^{23}$ de la main gauche en guise de drapeau, qui finalement ne sera pas annexée, et de Neuilly, qui ne le sera que partiellement, en dépit des vœux exprimés par un certain nombre de ses habitants ${ }^{24}$.

Le dessinateur montre des communes effrayées, qui crient et tentent de fuir car Paris essaie de les piéger en s'avançant sans bruit, les pieds nus, vers elles. La capitale cherche à les mettre en cage, à les emprisonner dans le cerceau de sa crinoline pour leur imposer son octroi. En courant pour s'enfuir, Auteuil tombe et se retrouve à quatre pattes. Dans sa précipitation, il a perdu la pancarte qu'il brandissait en signe de protestation. Ce mouvement de fuite des communes montre clairement qu'elles ne sont pas consentantes. Le comportement de la commune de Paris est lui aussi bien différent de celui décrit par le dessin qui paraîtra une fois l'agrandissement réalisé. C'est une ville conquérante, menaçante et prédatrice, et non plus à l'attitude bienfaisante, qui est ici dépeinte ${ }^{25}$. Dans ce nouveau contexte, la différence de tailles entre les personnages prend un tout autre sens que dans la première caricature. Elle traduit cette fois un rapport de forces entre d'une part une commune puissante et dominatrice et d'autre part des communes agressées qui ne sont pas de taille à lutter.

En dépit de l'existence de consultations préalables, la réforme prononcée par la loi du 16 juin 1859 fut assurément le résultat d'une décision autoritaire de l'empereur et de son préfet. À chaud, les témoins de l'événement pouvaient avoir des doutes quant aux intentions véritables de l'État et de la Ville de Paris à l'égard des territoires annexés. L'observation de la localisation des travaux effectués sous le Second Empire, situés avant tout au centre et à l'ouest de la capitale agrandie, montre qu'Haussmann n'avait pas renoncé à sa vision du Grand Paris de 1856, l'annexion ayant de surcroît immédiatement procuré à la Ville un

\footnotetext{
${ }^{20}$ Mémoire présenté par M. le Sénateur, préfet de la Seine, à la commission départementale faisant fonctions de Conseil général du département : session ordinaire de 1858, Paris, Charles Mourgues frères, 1859.

${ }^{21}$ C'est ce que montrent clairement les débats de la commission administrative de 1856. Voir Commission des circonscriptions..., op. cit.

${ }^{22}$ N. Montel, Le projet de Grand Paris..., op. cit.

${ }^{23}$ Palette de bois avec laquelle les blanchisseurs battent le linge. La commune de Boulogne abritait au XIX siècle de nombreuses blanchisseuses, qui lavaient le linge sur les bords de Seine, l'eau du fleuve en amont de la capitale étant réputée plus propre.

${ }^{24}$ AN, F² II Seine 37, dossier Neuilly.

${ }^{25}$ Dans une veine d'inspiration comparable, Cham représente à la même époque Paris en ogresse dévorant des enfants : "L'ogresse les avalant à mesure qu'ils grandissent (Clichy, Belleville, Batignolles, Nanterre) », Le Charivari, 28 novembre 1858.
} 
accroissement de ressources financières pour sa réalisation. En inscrivant distinctement le mot « octroi » sur le dernier arceau d'un jupon transformé en instrument de capture, c'est bien le ressort principal de la réforme que le dessinateur choisit en 1858 de mettre en lumière. Si, à partir du $1^{\text {er }}$ janvier 1860 , les populations annexées participent toutes au financement des travaux qui s'exécutent dans la capitale, seule une petite fraction voit sa contribution s'accompagner les années suivantes d'une amélioration de son cadre de vie. Cette situation alimente chez les laissés-pour-compte une rancœur qui ne tardera pas à trouver une traduction politique. 